\title{
Synthesis and characterization of the photoswitchable poly(methyl methacrylate-random-methacrylate spirooxazine)
}

\author{
Tam Huu Nguyen ${ }^{1}$, Phuc Huynh Tran², Linh Duy Thai ${ }^{2}$, Thuy Thu Truong ${ }^{1}$, Le-Thu T. Nguyen ${ }^{1}$ and \\ Ha Tran Nguyen ${ }^{1,3 *}$ (D)
}

\author{
'Faculty of Materials Technology, Ho Chi Minh City University of Technology - HCMUT, Vietnam National \\ University, Ho Chi Minh City, Vietnam \\ ${ }^{2}$ Faculty of Chemical Technology, Ho Chi Minh City University of Technology - HCMUT, Vietnam National \\ University, Ho Chi Minh City, Vietnam \\ ${ }^{3}$ National Key Laboratory of Polymer and Composite Materials, Vietnam National University, Ho Chi Minh \\ City, Vietnam \\ *nguyentranha@hcmut.edu.vn
}

\begin{abstract}
The photoswitchable poly(methyl methacrylate-random-methacrylate spirooxazine) was synthesized via atom transfer radical polymerization with the feed mole ratio of MMA/MSp comonomer of about 5.5/1. Well-defined poly(methyl methacrylate-random-methacrylate spirooxazine) have been obtained with the average molecular weight (Mn) of $6500 \mathrm{~g} / \mathrm{mol}$ and polydispersity of 1.21 . The structure and properties of the resulting copolymers were characterized by proton nuclear magnetic resonance (1H NMR), gel permeation chromatography, Fourier Transform infrared, UV-visible spectroscopy, and differential scanning calorimetry. Moreover, the copolymer exhibited the erasable and rewritable photoimaging on the solid state film which could to be as potential candidate for optical data storage materials.
\end{abstract}

Keywords: spirooxazine, controlled radical polymerization, photoswitching polymers.

How to cite: Nguyen, T. H., Tran, P. H., Thai, L. D., Truong, T. T., Nguyen, L.-T. T., \& Nguyen, H. T. (2019). Synthesis and characterization of the photoswitchable poly(methyl methacrylate-random-methacrylate spirooxazine). Polimeros: Ciência e Tecnologia, 29(1), e2019001. https://doi.org/10.1590/0104-1428.01118

\section{Introduction}

The creation of functional optical materials having photoactive properties has become one of the most promising objects in materials science. These materials are used in the fabrication of several optoelectronic devices such as optical memories, switches, and holograms. These integrated systems are basically formed by two components: the support media and the photoactive material, most of them are polymers functioned with photoactive molecules ${ }^{[1]}$. Photochromism has attracted much attention recently from the viewpoint of optical applications because of interest in refractive index or absorbance changes through optical extraction.

Since the last decade, the development of such optoelectronic devices has included the photochromic compounds as the active ingredient $t^{[1]}$. The photochromism of spiropyrans (SP) was reported by Fischer and Hirshberg ${ }^{[2]}$ in 1952 and since these organic compounds have been extensively studied due to their possible application in many fields. Following, spirooxazine compounds synthesized belonged to the spiroindolinonaphthoxazine ring system. Spirooxazine being a class of photochromic compounds are potentially applicable as chemical-UV-dosimeters for personal protection ${ }^{[3,4]}$ or they can be incorporated into the materials used for packaging applications in the case of UV sensitive products like food products. Generally, there is a great interest for the properties of photochromic compounds inserted into polymer films due to their possible use in practical applications. Spirooxazine are composed of an imide and a chromene moieties that are linked by a spirocarbon atom. Irradiation of spirooxazine with UV light induces heterolytic cleavage of the spiro-carboneoxygen bond, thus, producing the ring opened form, the intensively coloured merocyanine (MC). Merocyanine returns to the initial spirooxazine form in the dark or by visible light irradiation $^{[5-7]}$. The time to resume the initial colour depends on temperature and on the nature of the compound. In recent years, photochromic and thermochromic spiropyrans and spiroxazines have been receiving considerable attention, due to their potential application in many new technologies, such as data recording and storage, optical switching, displays, and non-linear optics ${ }^{[8-12]}$. Recently, Ventura et. al have reported the synthesis of new and well-defined poly(6-benzospiropyran hexylmethacrylate)s bearing a BSP moiety on the side chain of each unit of the polymer with linear, star-like and molecular brush architectures and narrow molecular weight distributions were successfully synthesized combining ATRP and click chemistry ${ }^{[13]}$. More 
recently, Dübner et. al have published the copolymer brushes, composed of glycidyl methacrylate and a furanprotected maleimide-containing monomer, after postpolymerization modification, they have functioned microperoxidase- 11 and photochromic spiropyran moieties, the polymer brushes catalyzed the oxidation of 3,3'5,5'- tetramethylbenzidine. Their obtained brushed copolymers exhibited the light-induced spiropyran-merocyanine transition under UV or visible-light, turnover by more than 1 order of magnitude ${ }^{[14]}$.

Here we reported the synthesis of poly(methyl methacrylate)-r-poly(methacrylate spirooxazine) (PMMA-rPMSp) via atom transfer radical polymerization (ATRP). The well- defined copolymers were characterized via $1 \mathrm{H}$ NMR, GPC, FTIR, DSC, UV-vis spectrum. Especially, the synthesized copolymers exhibited the spiroozazine-merocyanine transition under UV irradiation for both solution and solid film state.

\section{Materials and Methods}

\section{1 Materials}

2,7-Dihydroxynaphthalene (97\%), sodium nitrite $\left(\mathrm{NaNO}_{2}\right)$, sodium hydroxide $(\mathrm{NaOH})$, sulfuric acid $\left(\mathrm{H}_{2} \mathrm{SO}_{4}\right)$, hydrochloric acid $(\mathrm{HCl})$, potassium carbonate $\left(\mathrm{K}_{2} \mathrm{CO}_{3}\right)$, 1,3,3-trimethyl-2-methyleneindoline (97\%), triethylamine (99\%), methacryloyl chloride (97\%), Copper(I) bromide (CuBr, 98\%), $N, N, N^{\prime}, N^{\prime \prime}, N^{\prime \prime}$-pentamethyldiethylenetriamine (PMDETA, 99\%), ethyl $\alpha$-bromoisobutyrate (Et $t_{2} \mathrm{BriB}$, 98\%), 4-(Dimethylamino)pyridine (99\%) were purchased from Aldrich. Methyl methacrylate (MMA, 98\%) was purchased from Sigma-Aldrich passed through a plug of basic alumina before use in order to remove the hydroquinone inhibitors and stored under nitrogen atmosphere. Dichloromethane (99.8\%) and tetrahydrofuran (THF, 99\%) were purchased from Fisher/Acros and dried using molecular sieves under $\mathrm{N}_{2}$. Chloroform $\left(\mathrm{CHCl}_{3}, 99.5 \%\right)$, hexane (99\%), methanol (99.8\%), absolute ethanol (99\%) and ethyl acetate (99\%) were purchased from Fisher/Acros and used as received.

\subsection{Characterization}

${ }^{1} \mathrm{H}$ NMR spectra were recorded in deuterated chloroform $\left(\mathrm{CDCl}_{3}\right)$ with TMS $(\delta 0.00 \mathrm{ppm})$. The following abbreviations are used to describe the NMR signals: s (singlet), d (doublet), t (triplet), q (quartet), and br (broad). FT-IR spectra, collected as the average of 264 scans with a resolution of $4 \mathrm{~cm}^{-1}$, were recorded from $\mathrm{KBr}$ disk on the FT-IR Bruker Tensor 27. Size exclusion chromatography (SEC) measurements were performed on a Polymer PL-GPC 50 gel permeation chromatograph system equipped with an RI detector, with $\mathrm{THF}$ as the eluent at a flow rate of $1.0 \mathrm{~mL} / \mathrm{min}$. Molecular weight and molecular weight distribution were calculated with reference to polystyrene standards. UV-vis absorption spectra of polymers in solution and polymer thin films were recorded on a Shimadzu UV-2450 spectrometer over a wavelength range of $250-800 \mathrm{~nm}$.

\subsection{Synthesis of 1-nitrosonaphthalene-2,7-diol}

After dissolving $\mathrm{NaOH}(2.5 \mathrm{~g}, 62.4 \mathrm{mmol})$ in $100 \mathrm{~mL}$ of $\mathrm{H}_{2} \mathrm{O}, 2,7$-dihydroxynaphthalene (1) (10 g, $\left.62.4 \mathrm{mmol}\right)$ and $\mathrm{Na}_{2} \mathrm{NO}_{2}(4.3 \mathrm{~g}, 62.4 \mathrm{mmol})$ were added to the solution.
The reaction solution was heated for $1 \mathrm{~h}$ at $60{ }^{\circ} \mathrm{C}$ and then cooled to $0^{\circ} \mathrm{C}$. The mixture of $8 \mathrm{~mL}$ of concentrated $\mathrm{H}_{2} \mathrm{SO}_{4}$ and $15 \mathrm{~mL}$ of distilled water was added dropwise to the reaction solution with the temperature remained at $0{ }^{\circ} \mathrm{C}$. The reaction continued for $1 \mathrm{~h}$. After the reaction, the precipitate was isolated by vacuum filtration and washed with $0.1 \mathrm{M}$ aqueous $\mathrm{HCl}$ followed by cold water to obtain compound 2 as a dark brown powder solid. Yield: $11.22 \mathrm{~g}, 95 \%$.

${ }^{1} \mathrm{H}$ NMR, (500 MHz, methanol-d $), \delta(\mathrm{ppm}): 7.42(\mathrm{~d}, 1 \mathrm{H})$, $7.59(\mathrm{~d}, 1 \mathrm{H}), 7.56(\mathrm{~d}, 1 \mathrm{H}), 6.8(\mathrm{~d}, 1 \mathrm{H}), 6.18(\mathrm{~s}, 2 \mathrm{H})$. FT-IR $\left(\mathrm{cm}^{-1}\right): 3143(\mathrm{O}-\mathrm{H}), 1301(\mathrm{~N}=\mathrm{O})$.

\subsection{Synthesis of 1,3,3-trimethylspiro[indoline-2,3'- naphtho[2,1-b][1,4]oxazin]-9'-ol (spirooxazine-hydroxyl)}

To a suspension of 2,7-dihydroxy-1-nitrosonaphthaline (compound 1) (2.84 g, $15 \mathrm{mmol})$ in absolute ethanol $(50 \mathrm{~mL})$ was added dropwise, under refluxing a solution of 1,3,3-trimethyl-2-methyleneindoline (2.59 g, $2.65 \mathrm{~mL}$, $15 \mathrm{mmol})$ in absolute ethanol $(20 \mathrm{~mL})$. After continuous refluxing under a $\mathrm{N}_{2}$-stream, the obtained brown solution was purified over silica column with ethyl acetate/hexane (2:1) to obtain the crude product. Then, solvents were evaporated under vacuum to give a black powder. The black powder was washed with distilled water and extracted with $\mathrm{CHCl}_{3}$. Finally, the product was crystallized in hexane to obtain the pure white powder of SP. The product ( $2.74 \mathrm{~g}$, yield: $53 \%$ ) was dried in a vacuum oven at RT and was characterized by ${ }^{1} \mathrm{H}$ NMR and FT-IR.

${ }^{1} \mathrm{H}$ NMR, (500 MHz, $\left.\mathrm{CDCl}_{3}\right), \delta$ (ppm): 1.35 (s, 6H), $2.77(\mathrm{~s}, 3 \mathrm{H}), 6.58(\mathrm{t}, 1 \mathrm{H}), 6.84(\mathrm{~d}, 1 \mathrm{H}), 6.9(\mathrm{~d}, 1 \mathrm{H})$, $7.02(\mathrm{~d}, 1 \mathrm{H}), 7.09(\mathrm{~d}, 1 \mathrm{H}), 7.23(\mathrm{t}, 1 \mathrm{H}), 7.58(\mathrm{~d}, 1 \mathrm{H})$, $7.65(\mathrm{~d}, 1 \mathrm{H}), 7.71(\mathrm{~s}, 1 \mathrm{H}), 7.88(\mathrm{~s}, 1 \mathrm{H}), 10.04(\mathrm{~s}, 1 \mathrm{H}, \mathrm{OH})$. FT-IR $\left(\mathrm{cm}^{-1}\right)$ : $3313(\mathrm{O}-\mathrm{H}), 3065(=\mathrm{C}-\mathrm{H}), 1627(\mathrm{C}=\mathrm{N})$, $1301(\mathrm{~N}=\mathrm{O})$.

\subsection{Synthesis of the methacrylate spirooxazine monomer (MSp)}

Spirooxazine-hydroxyl (1.03 g, $3.00 \mathrm{mmol})$ was added to $25 \mathrm{~mL}$ of anhydrous dichloromethane in a $50 \mathrm{~mL}$ round bottomed flask. After cooling the solution to $0{ }^{\circ} \mathrm{C}$, triethylamine $(0.46 \mathrm{~g}, 0.627 \mathrm{~mL}, 4.5 \mathrm{mmol})$ was added and the reaction was stirred for an hour. Then, methacryloyl chloride $(0.47 \mathrm{~g}, 0.44 \mathrm{~mL}, 4.5 \mathrm{mmol})$ was dissolved in $5 \mathrm{~mL}$ of anhydrous dichloromethane and added dropwise to the reaction mixture under $\mathrm{N}_{2}$ atmosphere, cooled to $0{ }^{\circ} \mathrm{C}$. The reaction was continuously stirred for $24 \mathrm{~h}$ at room temperature. The solvent was rotary evaporated and the crude product was recrystallized in hexane. This crude product was purified over column silica hexane/ethyl acetate $(\mathrm{v}: \mathrm{v}=30 / 1)$ to obtain the pure white powder of Msp. The product was dried in a vacuum oven at RT and was characterized by ${ }^{1} \mathrm{H}$ NMR and FT-IR. Yield: 75\%.

${ }^{1} \mathrm{H}$ NMR, (500 MHz, $\left.\mathrm{CDCl}_{3}\right), \delta$ (ppm): 1.35 (s, 6H), $2.1(\mathrm{~s}, 3 \mathrm{H}), 2.77(\mathrm{~s}, 3 \mathrm{H}), 5.6(\mathrm{~s}, 1 \mathrm{H}), 6.3(\mathrm{~s}, 1 \mathrm{H}), 6.58(\mathrm{dd}, 1 \mathrm{H})$, $6.84(\mathrm{dd}, 1 \mathrm{H}), 6.9(\mathrm{~s}, 1 \mathrm{H}), 7.02(\mathrm{~d}, 1 \mathrm{H}), 7.09(\mathrm{~d}, 1 \mathrm{H})$, $7.23(\mathrm{~d}, 1 \mathrm{H}), 7.66(\mathrm{~d}, 1 \mathrm{H}), 7.72(\mathrm{~s}, 1 \mathrm{H}), 7.78(\mathrm{~d}, 1 \mathrm{H})$, $8.28(\mathrm{~d}, 1 \mathrm{H})$. FT-IR $\left(\mathrm{cm}^{-1}\right): 2971(=\mathrm{C}-\mathrm{H}), 1728(\mathrm{C}=\mathrm{O})$, $1627(\mathrm{C}=\mathrm{N}), 1121,740$. 


\subsection{Synthesis of Poly(methyl methacrylate-co- methacrylate spirooxazine) (Poly(MMA-co-MSp))}

Copolymer Poly(MMA-co-MSp) were synthesized using a ratio of [MMA]:[MSp]:[Et $\left.\mathrm{BriB}_{2}\right]:[\mathrm{CuBr}]:[\mathrm{PMDE}$ $\mathrm{TA}]=55: 10: 1: 1: 2$. Into a dried glass tube with a magnetic bar, $\mathrm{CuBr}(2.15 \mathrm{mg} \mathrm{g}, 0.015 \mathrm{mmol})$ and ligand PMDETA (26 mg, $0.15 \mathrm{mmol}$ ) were added, then monomer MMA ( $82.60 \mathrm{mg}, 0.75 \mathrm{mmol}), \mathrm{MSp}(61.87 \mathrm{mg}, 0.15 \mathrm{mmol})$, and anhydrous THF $(2 \mathrm{~mL})$ were added under nitrogen. The mixture was degassed by three freeze-pump-thaw cycles and purged with nitrogen. Et $\mathrm{BriB}_{2}(2.93 \mathrm{mg}, 0.015 \mathrm{mmol})$ was added before the polymerizations were carried out at $65{ }^{\circ} \mathrm{C}$ oil bath for $24 \mathrm{~h}$. The flask was then opened and exposed to air. The copolymer was recovered by removing the solvent under reduced pressure, re-dissolving the polymer in chloroform, passing the polymer solution through an alumina column to remove excess copper catalyst and was then concentrated by using a rotary evaporator. The concentrated solution was added in drops into an approximately $80 \mathrm{~mL}$ of cold methanol. The precipitation product was filtered through a medium frit funnel. Finally, the polymer was dried under vacuum at $60{ }^{\circ} \mathrm{C}$ for $24 \mathrm{~h}$ until a weight constant. Yield $75 \%, \mathrm{M}_{\mathrm{n}}=8000 \mathrm{~g} / \mathrm{mol}, \mathrm{M}_{\mathrm{w}} / \mathrm{M}_{\mathrm{n}}=1.34$, as obtained by gel permeation chromatography.

${ }^{1} \mathrm{H}$ NMR $\left(500 \mathrm{MHz}, \mathrm{CDCl}_{3}\right) \delta(\mathrm{ppm}): 0.8-1.3(\mathrm{~s}, 15 \mathrm{H})$, $1.35(\mathrm{~s}, 6 \mathrm{H}), 1.6-2.4(\mathrm{~s}, 4 \mathrm{H}), 2.76(\mathrm{~s}, 3 \mathrm{H}), 3.60(\mathrm{~s}, 3 \mathrm{H})$, $4.09(\mathrm{~m}, 2 \mathrm{H}), 6.58(\mathrm{dd}, 1 \mathrm{H}), 6.84(\mathrm{dd}, 1 \mathrm{H}), 6.9(\mathrm{~s}, 1 \mathrm{H})$, $7.02(\mathrm{~d}, 1 \mathrm{H}), 7.09(\mathrm{~d}, 1 \mathrm{H}), 7.23(\mathrm{~d}, 1 \mathrm{H}), 7.66(\mathrm{~d}, 1 \mathrm{H})$, $7.72(\mathrm{~s}, 1 \mathrm{H}), 7.78(\mathrm{~d}, 1 \mathrm{H}), 8.24(\mathrm{~d}, 1 \mathrm{H})$.

\section{Results and Discussions}

\subsection{Monomer synthesis}

First, 2,7-Dihydroxynaphthalene (compound 1) was nitrosated by $\mathrm{NaNO}_{2}$ in presence of $\mathrm{NaOH}$ and $\mathrm{H}_{2} \mathrm{SO}_{4}$ to obtain 1-nitrosonaphthalene-2,7-diol (compound 2, Scheme 1). Then, 1-nitrosonaphthalene-2,7-diol reacted with 1,3,3-trimethyl2-methyleneindoline to form spirooxazine-hydroxyl (compound 3, Scheme 1), which subsequently reacted with methacryloyl chloride to give methacrylate-spirooxazine
(MSp) (compound 4, Scheme 1). Finally, the synthesis of poly(MMA-co-MSp) (coumpound 5, Scheme 1) was performed via atom transfer radical polymerization using $\mathrm{CuBr}$ and PMDETA as catalyst system. The synthesis of poly(MMA-co-MSp) was described as Scheme 1.

The FTIR was used to characterize the spirooxazine hydroxyl (compound 3) and methacrylate spirooxazine (MSp) (compound 4) to determine the chemical functional groups in their structure. Figure 1 showed the FTIR of spirooxazine hydroxyl and methacrylate-spirooxazine, the vibration peaks at $1630 \mathrm{~cm}^{-1}$ corresponding to $\mathrm{N}=\mathrm{C}$ linkage and the broad peaks at $3300 \mathrm{~cm}^{-1}$ assigned to $\mathrm{OH}$ group in spirooxazine hydroxyl (green line c, Figure 1). Following, Spirooxazine-hydroxyl was reacted with methacryloyl chloride to give methacrylate-spirooxazine (MSp) in the presence of triethylamine as catalytic. The reaction was performed in 24 hours. The obtained product was purified over column using heptane/ethyl acetate as eluent, and the purified product was recrystallized in methanol in the yield of $75 \%$. The obtained methacrylate spirooxazine exhibited the vibration peaks at $1750 \mathrm{~cm}^{-1}$ corresponding to $\mathrm{C}=\mathrm{O}$ linkage, and the peaks of $\mathrm{OH}$ group at $3300 \mathrm{~cm}^{-1}$ disappears completely that confirmed that the esterification of spirooxazine hydroxyl with methacryloyl chloride was taken place successfully (cyan line d, Figure 1).

\subsection{Polymer synthesis}

The ${ }^{1} \mathrm{H}$ NMR spectrum of spirooxazine hydroxyl showed the proton resonance of imine linkage at $7.65 \mathrm{ppm}$. Moreover, the signals at $2.65 \mathrm{ppm}$ and $1.42 \mathrm{ppm}$ exhibited for the methyl groups of 1,3,3- trimethyl-2-methyleneindoline in sprirooxazine hydroxyl moieties. The ${ }^{1} \mathrm{H}$ NMR spectrum of MSp (Figure 2) showed the proton resonance of methylene linkage of methacrylate at $5.70 \mathrm{ppm}$ and $6.30 \mathrm{ppm}$. The ${ }^{1} \mathrm{H}$ NMR spectrum of MSp also showed the imine linkage in methacrylate - spirooxazine ring at $8.4 \mathrm{ppm}$ which was shifted from $7.65 \mathrm{ppm}$ in spirooxazine-hydroxyl. All other proton resonances appear in the reasonable intensities and are correlated to spirooxazine structure, thus confirming the expected molecular structure of MSp. The attribution

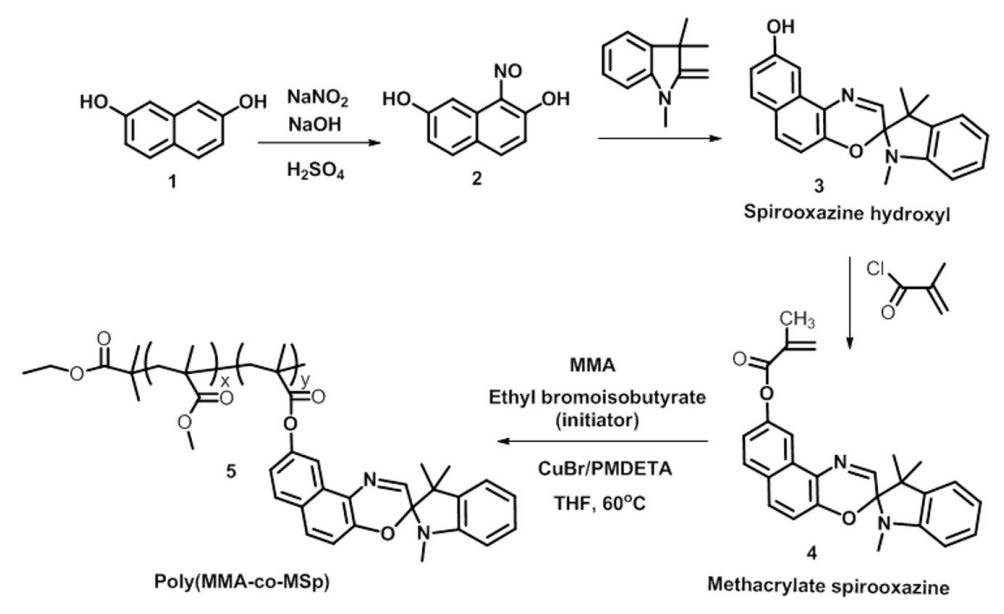

Scheme 1. Synthesis of poly(methyl methacrylate-co-methacrylate spirooxazine). 


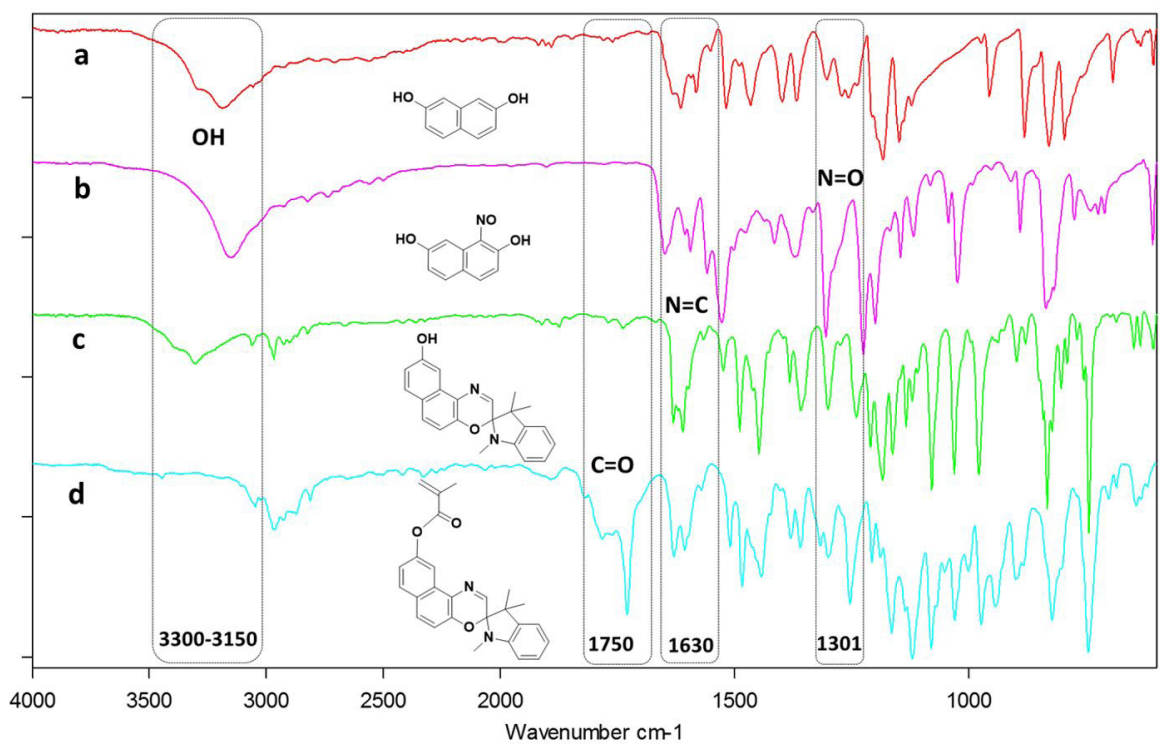

Figure 1. FTIR of monomer compounds: (a) FTIR of 2,7-dihydroxynaphthalene compound; (b) FTIR of 1-nitrosonaphthalene-2,7-diol compound; (c) FTIR of spirooxazine-hydroxyl; (d) FTIR of methacrylate spirooxazine monomer.

(A)

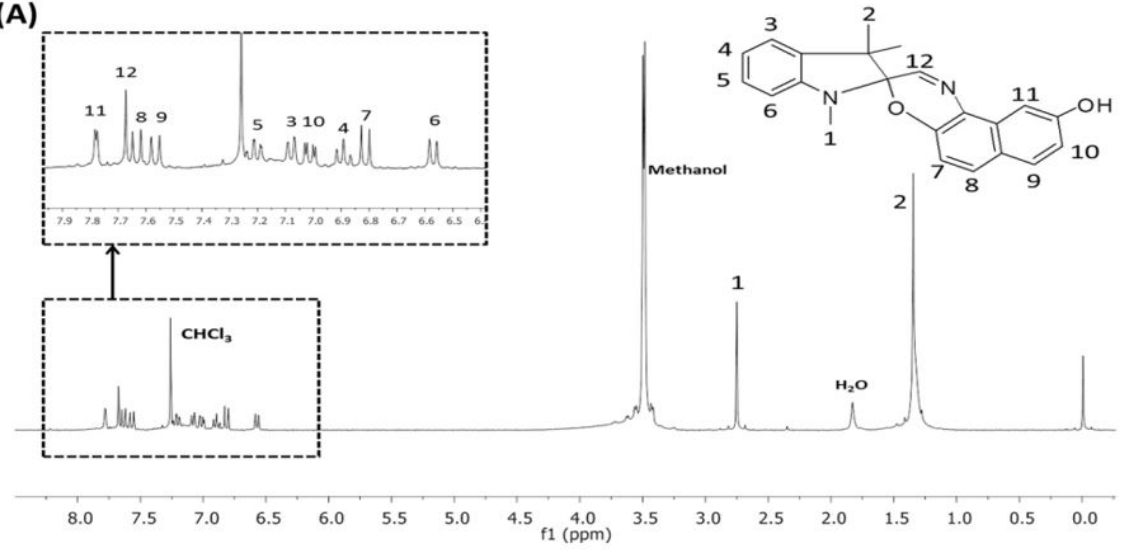

(B) $\mathrm{CHCl}_{3}$

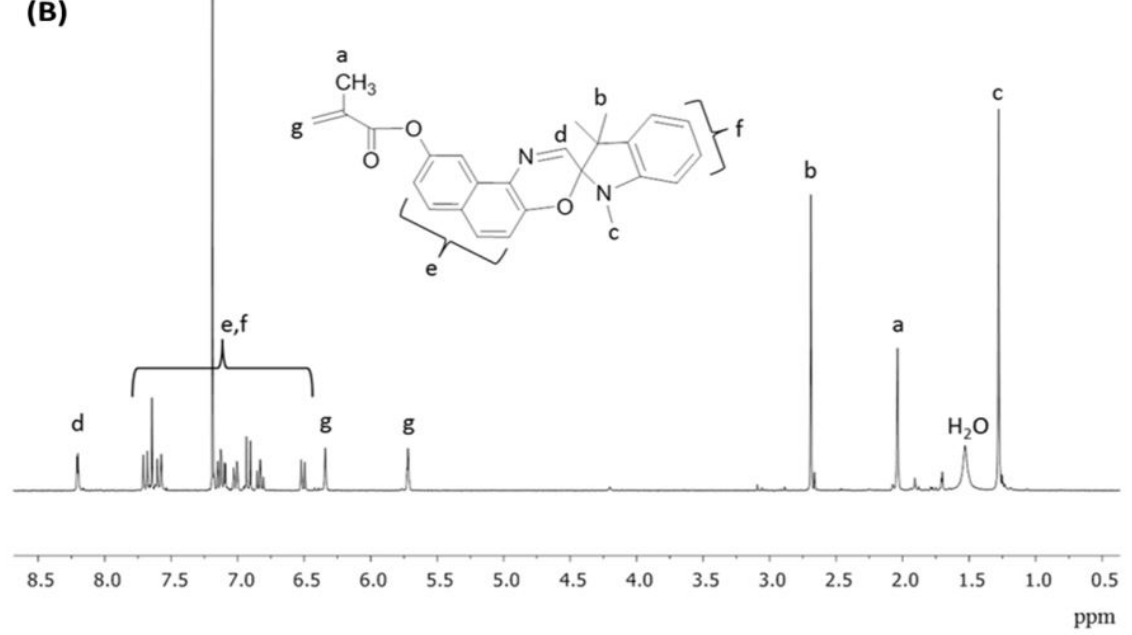

Figure 2. The ${ }^{1} \mathrm{H}$ NMR of spirooxazine hydroxyl and methacrylate spirooxazine (MSp): (A) ${ }^{1} \mathrm{H}$ NMR of spirooxazine hydroxyl; (B) ${ }^{1} \mathrm{H}$ NMR of methacrylate spirooxazine). 
of the ${ }^{1} \mathrm{H}$ NMR signals of spirooxazine hydroxyl and MSp is presented in Figure $2^{[15]}$.

In final step, the MSp and MMA comonomers were polumerized via ATRP using ethyl $\alpha$-bromoisobutyrate in the presence of $\mathrm{CuBr}$ and PMDETA as catalyst and ligand, respectively. The feed ratio of MMA/MSp comonomer of about 5.5/1 was established for achieving a good control over ATRP $([\mathrm{MMA}] /[\mathrm{MSp}] /[$ [nitiator] $] /[\mathrm{CuBr}] /[\mathrm{PMEDETA}]=$ $55: 5: 1: 1: 2)$. The polymerization was performed in THF at $60^{\circ} \mathrm{C}$ for $24 \mathrm{~h}$ under nitrogen atmosphere. The polymerization was stopped by cooling the reaction mixture followed by dilution with extra volume of THF, then the mixture was purified over aluminium column to remove $\mathrm{CuBr}$ catalyst. The copolymers were obtained by precipitation in cold $n$-heptane, the copolymers were filtered and dried under vacuum until constant mass.

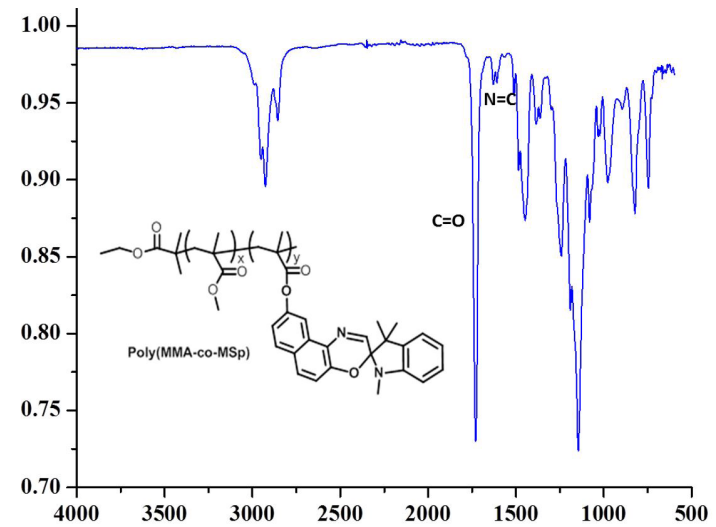

Figure 3. The FT-IR of copolymers Poly(MMA-r-MSp).
The FT-IR spectra was used to characterize the copolymers Poly(MMA-r-MSp) as Figure 3. The appearance of the high intensity signal observed at $1728 \mathrm{~cm}^{-1}$ that attributed to carbonyl vibrational $(\mathrm{C}=\mathrm{O})$ of Poly(MMA- $r$-MSp). In addition, the $\mathrm{N}=\mathrm{C}$ linkage of MSp in Poly(MMA- $r$-MSp) was also exhibited at $1651 \mathrm{~cm}^{-1}$.

The polymer structure of Poly(MMA- $r$-MSp) was also confirmed by ${ }^{1} \mathrm{H}$ NMR. The Figure 4 exhibited all characteristic peaks of copolymers Poly(MMA-r-MSp). The polymerization degree of the Poly(MMA-r-MSp) was calculated from recorded ${ }^{1} \mathrm{H}$ NMR spectrum by comparing the relative signal intensities of the imine proton of the MSp and methylene protons of the MMA residue at $\delta=8.24 \mathrm{ppm}$ (peak "s", Figure 4) and $\delta=3.6 \mathrm{ppm}$ (peak "f", Figure 4), respectively, with that of the methylene protons of initiator at $4.09 \mathrm{ppm}$ (peak "b", Figure 4). The molecular weight of the copolymers Poly(MMA- $r$-MSp) was determined to be $6500 \mathrm{~g} / \mathrm{mol}$ and to compise 52 and 3 of MMA and MSp units, respectively. These results give the weight compositions of $80 \%$ and $20 \%$ of MMA and MSp, respectively in copolymers Poly(MMA-r-MSp).

As seen from Table 1, the Poly(MMA-r-MSp) was obtained with a relatively good approximation between theoretical and experiment molar masses that approved for an initiation efficiency close to 1. A narrow molecular weight distribution of the copolymers Poly(MMA-r-MSp) was recorded by GPC, with $\mathrm{Ð}_{\mathrm{M}}=1.21$.

\subsection{Photoisomerization properties of polymers}

Photoisomerization property of poly(MMA- $r$-MSp) was studied in THF. Figure 5A and Figure 5B show the color changing of polymer solution $(0.1 \mathrm{~g} / \mathrm{L} ; \mathrm{pH} 9.0)$ measured at $25^{\circ} \mathrm{C}$ under UV irradication. Without UV irradiation, the

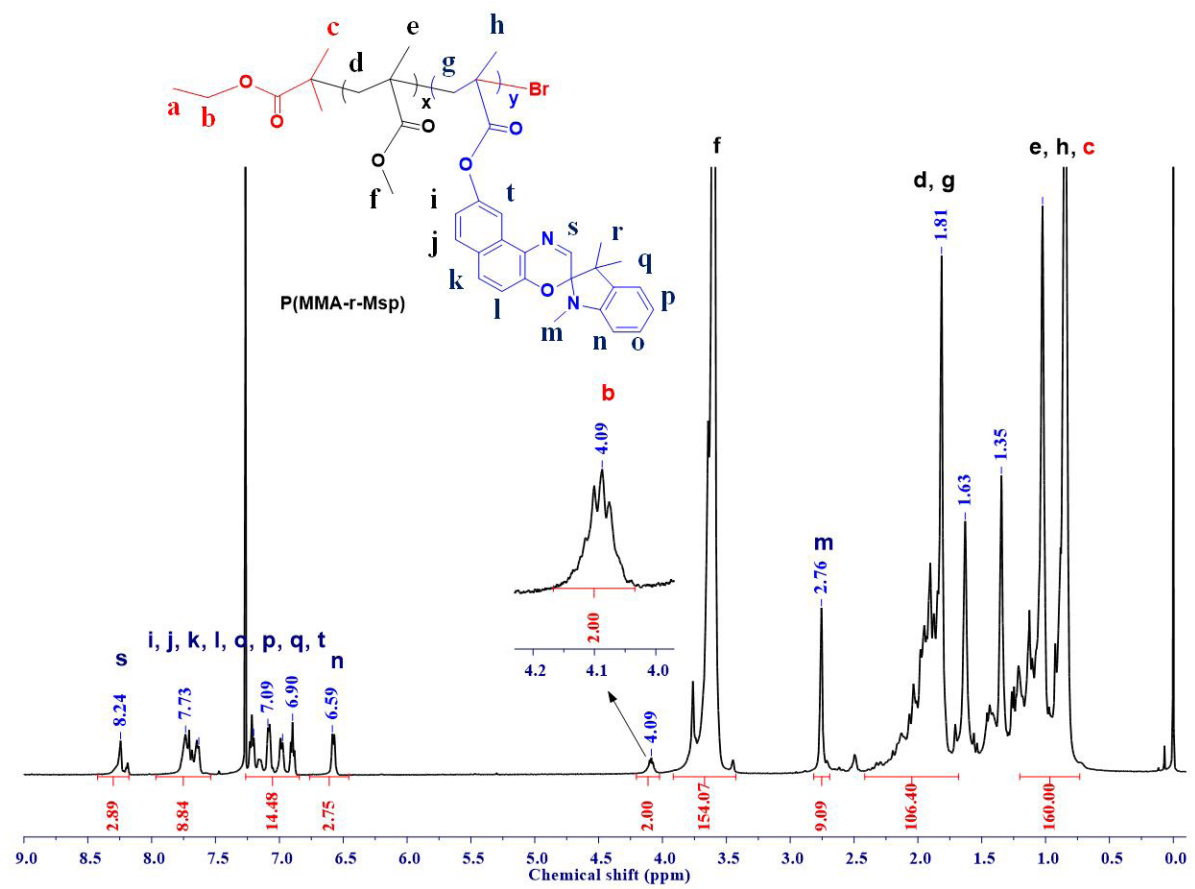

Figure 4. The ${ }^{1} \mathrm{H}$ NMR spectrum of copolymers Poly(MMA-r-MSp). 
Table 1. Macromolecular characteristics of Poly(MMA- $r$-MSp) synthesized by ATRP using $\alpha$-bromoisobutyrate initiator and CuBr/ $\operatorname{PMDETA}([\mathrm{CuBr}] /[\mathrm{PMDETA}]=1 / 2)$ as the catalytic complex.

\begin{tabular}{|c|c|c|c|c|c|c|c|c|c|}
\hline \multirow[b]{2}{*}{$\begin{array}{c}\text { Diblock } \\
\text { copolymers }\end{array}$} & \multirow[b]{2}{*}{$\mathbf{T}^{\circ} \mathbf{C}$} & \multirow[b]{2}{*}{$\begin{array}{c}\text { Conversion } \\
(\%)^{(a)}\end{array}$} & \multicolumn{2}{|c|}{ MMA } & \multicolumn{2}{|c|}{ MSp } & \multirow[b]{2}{*}{$\mathbf{f}^{(\mathrm{d})}$} & \multicolumn{2}{|c|}{ Poly(MMA-r-MSp) } \\
\hline & & & $\begin{array}{l}\bar{M}_{\mathrm{n} \text { the }}{ }^{(\mathrm{b})} \\
\text { (g/mol) }\end{array}$ & $\begin{array}{l}\bar{M}_{\mathrm{n} \exp }{ }^{(\mathrm{c})} \\
(\mathrm{g} / \mathrm{mol})\end{array}$ & $\begin{array}{l}\bar{M}_{\mathrm{n} \text { the }}{ }^{(\mathrm{b})} \\
(\mathrm{g} / \mathrm{mol})\end{array}$ & $\begin{array}{l}\bar{M}_{\mathrm{n} \exp }{ }^{(\mathrm{c})} \\
(\mathrm{g} / \mathrm{mol})\end{array}$ & & $\begin{array}{l}\bar{M}_{\text {n MMr }} \\
\text { (g/mol) }\end{array}$ & $\boldsymbol{Ð}^{(\mathrm{e})}$ \\
\hline 1 & 60 & 92 & 5060 & 5200 & 2150 & 1300 & 1 & 6500 & 1.21 \\
\hline 2 & 60 & 87 & 4785 & 5100 & 2150 & 1300 & 1 & 6400 & 1.25 \\
\hline
\end{tabular}

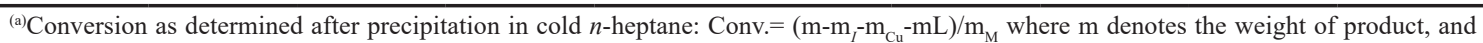
$\mathrm{m}_{\mathrm{I}}, \mathrm{m}_{\mathrm{Cu}}, \mathrm{mL}, \mathrm{m}_{\mathrm{M}}$ the weights of the initiator, copper catalyst, ligand (PMDETA), and monomers, respectively; (b)MMA and MSp theoretical number-average-molar mass as calculated by [MMA] or [MSp) $]_{0} /[\text { Initiator }]_{0} \times \operatorname{Conv}(\%) \times \mathrm{M}_{\mathrm{wMMA}(\text { or MSp) }}$ assuming a living process; (c) MMA(or MSp) experimental number-average molar mass as determined by ${ }^{1} \mathrm{H}$ NMR spectroscopy (see Figure 3 ): $\bar{M}_{\mathrm{nexp}}=D P_{E X P} x M_{W M M A(O R M S P)}$ where $D P_{E X P}$ is the experimental degree of polymerization, as calculated from the relative intensities of methylene protons of $\mathrm{MMA}(\delta=3.6 \mathrm{ppm})$, imine proton of

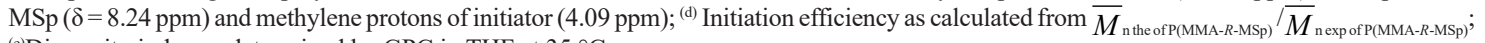
${ }^{(e)}$ Dispersity index as determined by GPC in THF at $35^{\circ} \mathrm{C}$.

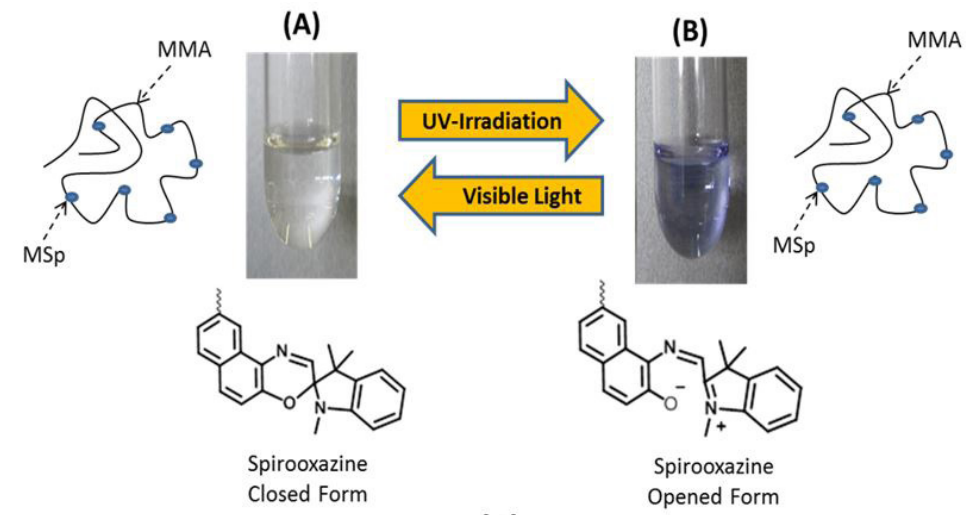

(C)

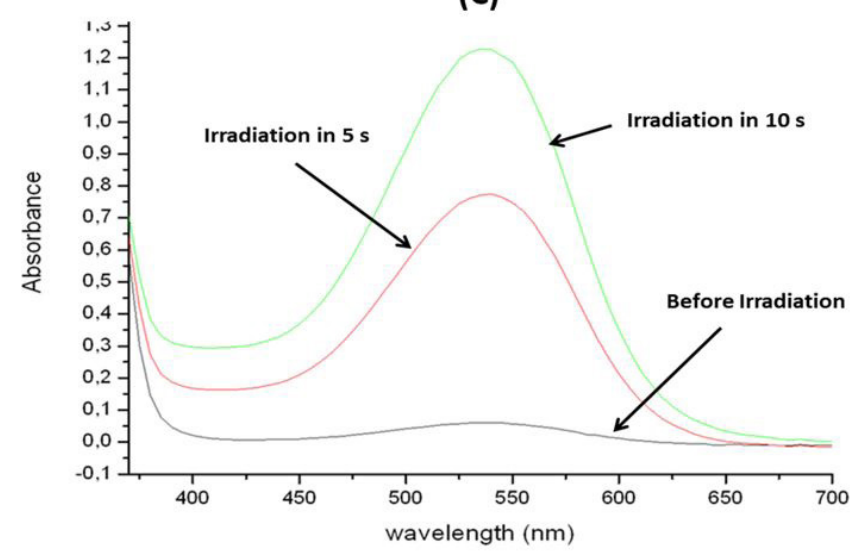

Figure 5. Poly(MMA-r-MSp) in THF solutionwith MSp in closed form (A), Poly(MMA-r-MSp) in THF solution with MSp in opened form after UV irradication (B), UV-Vis spectra of poly(MMA-r-MSp) under UV-irradiation (C).

polymer shows almost no absorption in the visible region (blue line, Figure 5C) indicating that the spirooxazine units exist as a closed form, as shown in Figure 5C. However, UV irradiation of the solution $(334 \mathrm{~nm})$, creates a distinctive absorption band centered at $540 \mathrm{~nm}$, assigned to the generation of opened form of spirooxazine.

In addition, we measured the absorption spectra changes of poly(MMA-r-MSp) to obtain an insight into their photochromic properties in the solid state film which shows similarly photochromic performance in solution. The copolymers poly(MMA-r-MSp) solution was coated on transparent glass substrate via spin coating method, then the obtained polymer film was dried in oven at $60{ }^{\circ} \mathrm{C}$ in 2 hours . Irradiation a colorless poly(MMA- $r$-MSp) film, the colorless poly(MMA-r-MSp) turned to blue and a new absorption band appears at around $550 \mathrm{~nm}$ and gradually increased and reached a photostationary state.

The inherent characteristics of poly(MMA- $r$-MSp) film make it possible to use such materials for data recording. A possible procedure for data recording and erasing is presented in Figure 6. Upon UV light irradiation through the mask, the optical data were recorded on poly(MMA-r-MSp) film irradiation region, when irradiation with visible light on irradiation region, the optical data were erased. In solid 

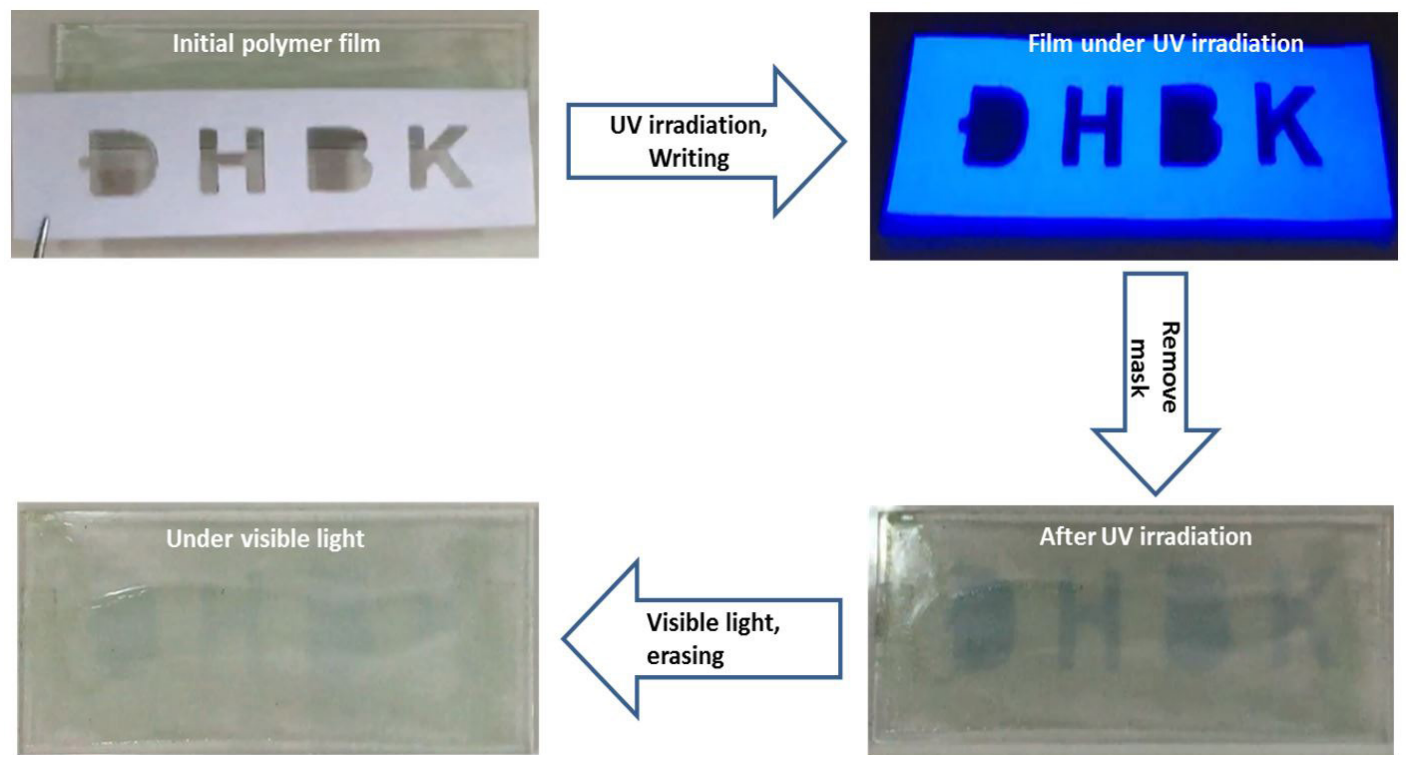

Figure 6. Principle scheme of the optical data recording on poly(MMA-r-MSp) film.

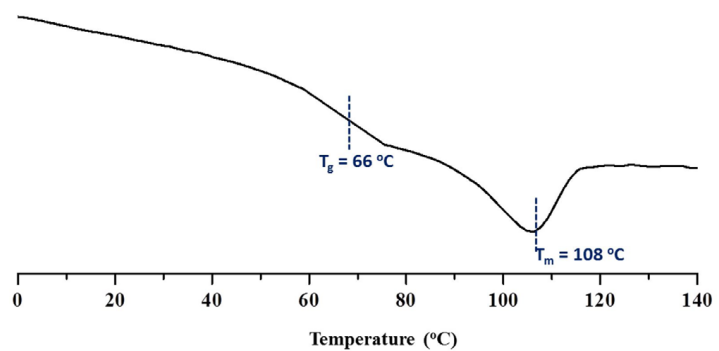

Figure 7. The DSC diagram of poly(MMA-r-MSp).

state, the practical capability of rewritable photoimaging on solid state investigated by patterned illumination through photomasks. The word 'ĐHBK' (Ho Chi Minh City University of Technology, Vietnamese abbreviated) was recorded as a first image (Figure 6), which was subsequently erased and followed by the recording of a second image. The cycles of writing and erasing was repeated more than 30 times.

Last but not least, the thermal properties of poly(MMA- $r$-MSp) $\left(M_{n}=6500 \mathrm{~g} / \mathrm{mol}, Ð=1.21\right)$ was characterized to determine the glass transition of copolymers by DSC. The DSC second-heating traces in the range from 0 to $150{ }^{\circ} \mathrm{C}$ of the poly(MMA-r-MSp) are shown in Figure 7. For such studied molecular weight, the glass transition temperature $\left(\mathrm{T}_{\mathrm{g}}\right)$ value of poly(MMA- $r$-MSp) is around $66{ }^{\circ} \mathrm{C}$. Otherwise, the melting point $\left(\mathrm{T}_{\mathrm{m}}\right)$ of poly(MMA-r-MSp) is observed at ca. $108{ }^{\circ} \mathrm{C}$ which is similar to the DSC results of pure homopolymer PMMA in the same of average molecular weight.

\section{Conclusions}

In conclusion, we have successfully designed and synthesized a novel photoswitching poly(MMA- $r$-MSp) via controlled polymerization method. The obtained copolymer poly(MMA-r-MSp) has the average molecular weight of $6500 \mathrm{~g} / \mathrm{mol}$ with polydispersity index of around 1.2-1.3. The copolymer exhibited excellent photochromic behavior in solid film under UV irradiation. Erasable and rewritable photoimaging on the solid film was successfully demonstrated. This optical data storage materials based on photochromic poly(MMA-r-MSp) could to be as candidate for fundamental studies and eventual technical application for all-photo mode - high density optical data.

\section{Acknowledgements}

The study was supported by Science and Technology Incubator Youth Program, managed by the Center for Science and Technology Development, Ho Chi Minh Communist Youth Union, the contract number is “15/2017/HĐ-KHCN-VU' ", and project "C2017-20-31" from Ho Chi Minh City University of Technology - Vietnam National University - Ho Chi Minh City, 268 Ly Thuong Kiet, District 10, Ho Chi Minh City, Viet Nam.

\section{References}

1. Mizokuro, T., Mochizuki, H., Kobayashi, A., Horiuchi, S., Yamamoto, N., Tanigaki, N., \& Hiraga, T. (2004). Selective doping of photochromic dye into nanostructures of diblock copolymer films by vaporization in a vacuum. Chemistry of Materials, 16(18), 3469-3475. http://dx.doi.org/10.1021/ $\mathrm{cm} 049557 \mathrm{u}$.

2. Fischer, E., \& Hirshberg, Y. (1954). Photochromism and reversible multiple internal transitions in some spiropyrans at low temperatures. Journal of the Chemical Society, 297-303. http://dx.doi.org/10.1039/JR9540000297.

3. Lokshin, V. A., Samat, A., \& Metelitsa, A. V. (2002). Spirooxazines: synthesis, structure, spectral and photochromic properties. Russian Chemical Reviews, 71(11), 893-916. http:// dx.doi.org/10.1070/RC2002v071n11ABEH000763. 
4. Bouas-Laurent, H., \& Dürr, H. (2001). Organic photochromism. Pure and Applied Chemistry, 73(4), 639-665. http://dx.doi. org/10.1351/pac200173040639.

5. Berkovic, G., Krongauz, V., \& Weiss, V. (2000). Spiropyrans and spirooxazines for memories and switches. Chemical Reviews, 100(5), 1741-1754. http://dx.doi.org/10.1021/cr9800715. PMid: 11777418.

6. Lin, J. S. (2003). Interaction between dispersed photochromic compound and polymer matrix. European Polymer Journal, 39(8), 1693-1700. http://dx.doi.org/10.1016/S0014-3057(03)00058-2.

7. Ock, K., Jo, N., Kim, J., Kim, S., \& Koh, K. (2001). Thin film optical waveguide type UV sensor using a photochromic molecular device, spirooxazine. Synthetic Metals, 117(1-3), 131-133. http://dx.doi.org/10.1016/S0379-6779(00)00553-1.

8. Myles, A. J., Wigglesworth, T. J., \& Branda, N. R. (2003). A multi-addressable photochromic 1,2-dithienylcyclopentenephenoxynaphthacenequinone hybrid. Advanced Materials, 15(9), 745-748. http://dx.doi.org/10.1002/adma.200304917.

9. Corredor, C. C., Huang, Z. L., \& Belfield, K. D. (2006). Twophoton $3 \mathrm{D}$ optical data storage via fluorescence modulation of an efficient fluorene dye by a photochromic diarylethene. Advanced Materials, 18(21), 2910-2914. http://dx.doi. org/10.1002/adma.200600826.

10. Lim, S. J., Seo, J., \& Park, S. Y. (2006). Photochromic switching of excited-state intramolecular proton-transfer (ESIPT) fluorescence: a unique route to high-contrast memory switching and nondestructive readout. Journal of the American Chemical Society, 128(45), 14542-14547. http://dx.doi.org/10.1021/ ja0637604. PMid:17090038.
11. Lim, S. J., An, B. K., \& Park, S. Y. (2005). Bistable photoswitching in the film of fluorescent photochromic polymer: enhanced fluorescence emission and its high contrast switching. Macromolecules, 38(15), 6236-6239. http://dx.doi.org/10.1021/ ma0504163.

12. Jiang, G., Wang, S., Yuan, W., Jiang, L., Song, Y., Tian, H., \& Zhu, D. (2006). Highly fluorescent contrast for rewritable optical storage based on photochromic bisthienylethene-bridged naphthalimide dimer. Chemistry of Materials, 18(2), 235-237. http://dx.doi.org/10.1021/cm052251i.

13. Ventura, C., Thornton, P., Giordani, S., \& Heise, A. (2014). Synthesis and photochemical properties of spiropyran graft and star polymers obtained by 'click' chemistry. Polymer Chemistry, 5(21), 6318-6324. http://dx.doi.org/10.1039/C4PY00778F.

14. Qu, W.-J., Li, W.-T., Zhang, H.-L., Wei, T.-B., Lin, Q., Yao, H., \& Zhang, Y.-M. (2017). A rational designed fluorescent and colorimetric dual-channel sensor for cyanide anion based on the PET effect in aqueous medium. Sensors and Actuators. B, Chemical, 241, 430-437. http://dx.doi.org/10.1016/j. snb.2016.10.100.

15. Kim, S. H., Hwang, I. J., Gwon, S. Y., \& Son, Y. A. (2010). Photoregulated optical switching of poly(N-isopropylacrylamide) hydrogel in aqueous solution with covalently attached spironaphthoxazine and D- $\pi$-A type pyran-based fluorescent dye. Dyes and Pigments, 87(2), 158-163. http://dx.doi. org/10.1016/j.dyepig.2010.03.014.

Received: Feb. 10, 2018 Revised: Apr. 16, 2018 Accepted: Apr. 28, 2018 